\title{
Sauvetage: une prise en charge optimale grâce au système dual
}

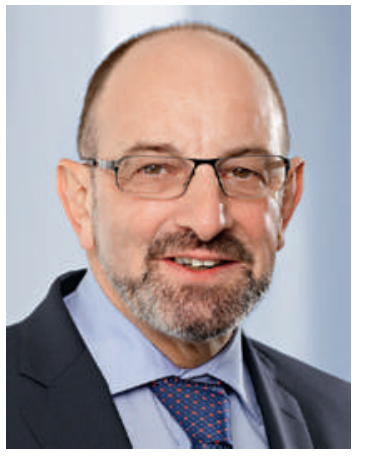

Souvent considérés comme un indice de qualité pour la prise en charge médicale de la population, les soins d'urgence et le sauvetage reposent sur l'implication de professionnels de la santé issus de domaines très différents qui interagissent à l'instar des roues dentées d'un engrenage, garantissant des soins optimaux tout au long de la chaîne du sauvetage.

Le Forum du sauvetage de la FMH constitue une plateforme d'échange entre les sociétés de discipline médicale participant à l'assistance préhospitalière d'urgence; c'est pourquoi les représentants médicaux de l'Interassociation de sauvetage (IAS) et du Service sanitaire coordonné de l'armée y sont également présents. Les douze thèses sur le sauvetage qui ont contribué de façon marquante au développement et à la professionnalisation du système helvétique de sauvetage ont été remplacées en août 2010 par les nouvelles lignes directrices de la FMH pour le sauvetage. Fortes de nouvelles perspectives, ces lignes directrices visent à uniformiser l'assistance professionnelle d'urgence dans toute la Suisse, également en cas de catastrophe et autres événements graves, et à lui conférer un caractère obligatoire. Par ailleurs, la FMH s'engage pour que les médecins d'urgence ou les médecins de famille spécialement formés (médecins de garde) assurent les soins d'urgence conjointement avec les ambulanciers.

\section{Ambulanciers et médecins d'urgence / médecins de garde agissent conjointement lors de sauvetages et de situations d'urgence.}

Avec l'élaboration et l'édition des lignes directrices en 2010, le Forum du sauvetage a contribué à actualiser la stratégie de la FMH en la matière. Il a mené des entretiens avec les acteurs usuels du sauvetage et il a aussi encouragé la publication d'articles relatant les activités entreprises aux différents niveaux de la chaîne des soins médicaux urgents. Nous avons d'un côté les urgentistes ou médecins de garde et de l'autre les ambulanciers qui conjuguent leurs efforts pour garantir la prise en charge des soins d'urgence tout au long de la chaîne du sauvetage. La FMH se félicite de la mise en place de ce «système dual» pour assurer l'assistance d'urgence. Il permet en effet - dans ce qu'on appelle communément un système de «rendez-vous» - que les ambulanciers interviennent avec l'aide soit d'un médecin d'urgence soit d'un médecin de garde. Pour pérenniser ce système dual, il faut une volonté politique mais aussi des réglementations cantonales qui le portent et le soutiennent de manière adéquate.

Les premiers soins médicaux, que prodiguent les services helvétiques d'assistance médicale d'urgence au sol, se sont continuellement améliorés au cours de ces dix dernières années grâce, notamment, à des formations aux métiers du sauvetage régulièrement adaptées en conséquence. En 35 ans, le métier d'ambulancier a évolué pour donner lieu à la création de deux certificats distincts. La première volée d'ambulanciers a été diplômée en 1977, l'Association suisse des ambulanciers (ASA) a vu le jour en 1999, et la nouvelle loi sur la formation professionnelle qui réglemente toutes les professions, et donc aussi les professions paramédicales, est entrée en

\section{Le système dual des soins urgents doit être le fruit d'une volonté politique et bénéficier d'un soutien adéquat des cantons.}

vigueur en 2002. Le transfert des compétences à l'Office fédéral de la formation professionnelle et de la technologie (OFFT) a permis de définir les conditions-cadres et de mettre en place la formation pour le diplôme fédéral d'ambulancier ES. Grâce à l'uniformisation de la formation, les compétences médicales revêtent désormais nettement plus d'importance que par le passé. Cependant, cette possibilité donnée aux médecins de déléguer davantage de tâches médicales aux ambulanciers crée des différences régionales: principalement dans les régions isolées, les services de sauvetage sans système de médecins d'urgence ont tendance à disposer d'un domaine de compétence plus large pour intervenir.

Les différentes organisations médicales et paramédicales œuvrent ensemble pour garantir que les personnes gravement atteintes dans leur santé ou accidentées soient prises en charge de manière optimale par du personnel expérimenté, et transportées à l'hôpital avec des moyens de sauvetage adéquats.

Le Forum a appelé de ses vœux une série consacrée au sauvetage qui se poursuit dans ce numéro avec l'article «Ambulancier, une profession en pleine mutation» (cf. page 687).

Dr Ernst Gähler, vice-président de la FMH, responsable du domaine Professions paramédicales 